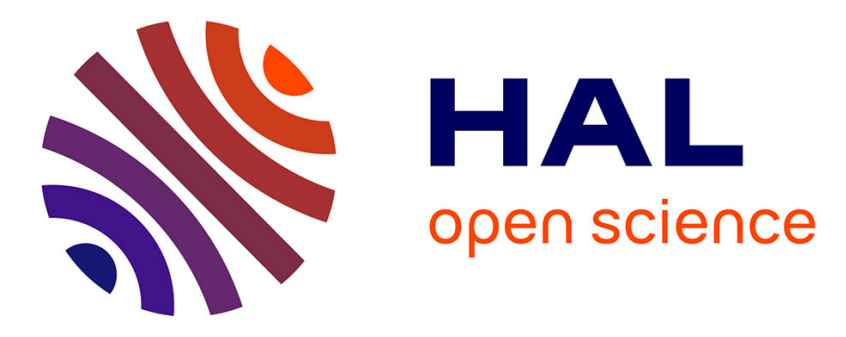

\title{
Comparison of registration methods for mobile manipulators
}

\author{
Roger Bostelman, Roger Eastman, Tsai Hong, Omar Aboul Enein, Steven \\ Legowik, Sebti Foufou
}

\section{To cite this version:}

Roger Bostelman, Roger Eastman, Tsai Hong, Omar Aboul Enein, Steven Legowik, et al.. Comparison of registration methods for mobile manipulators. 19th International Conference on Clawar 2016 , Sep 2016, London, United Kingdom. pp.205 - 213, 10.1142/9789813149137_0026 . hal-01401564

\section{HAL Id: hal-01401564 https://hal.science/hal-01401564}

Submitted on 27 Nov 2016

HAL is a multi-disciplinary open access archive for the deposit and dissemination of scientific research documents, whether they are published or not. The documents may come from teaching and research institutions in France or abroad, or from public or private research centers.
L'archive ouverte pluridisciplinaire HAL, est destinée au dépôt et à la diffusion de documents scientifiques de niveau recherche, publiés ou non, émanant des établissements d'enseignement et de recherche français ou étrangers, des laboratoires publics ou privés. 


\title{
COMPARISON OF REGISTRATION METHODS FOR MOBILE MANIPULATORS
}

\author{
ROGER BOSTELMAN ${ }^{1,2}$, ROGER EASTMAN ${ }^{3}$, TSAI HONG ${ }^{1}$, \\ OMAR ABOUL ENEIN ${ }^{1}$, STEVEN LEGOWIK ${ }^{4}$, SEBTI FOUFOU \\ ${ }^{1}$ National Institute of Standards and Technology, Engineering Laboratory, Intelligent \\ Systems Division, Gaithersburg, MD, USA \\ ${ }^{2}$ Le2i Lab, Université de Bourgogne, BP 47870, 21078 Dijon, France \\ ${ }^{3}$ Loyola University Maryland, Baltimore, MD, USA \\ ${ }^{4}$ Robotic Research, LLC, Gaithersburg, MD, USA \\ ${ }^{5}$ CSE Dept, College of Engineering, Qatar University, Qatar.
}

\begin{abstract}
Mobile manipulators can be effective, efficient and flexible for automation on the factory floor but will need safety and performance standards for wide adoption. This paper looks at a specific area of performance standards [1] for docking and workpiece registration, with the intent of evaluating how quickly, repeatably, and accurately a mobilemanipulator end effector can be aligned with a known physical target to facilitate peg-in-hole insertion tasks. To evaluate mobile manipulator docking, we conducted experiments with an AGV-mounted arm in a laboratory space equipped with an extensive optical tracking system and a standardized test piece (artifacts) simulating an industrial assembly. We experimented with different strategies and sensors for registration and report on these approaches.
\end{abstract}

\section{Introduction}

Mobile manipulators (i.e., robot arms onboard mobile robotic bases) hold promise in industrial applications ${ }^{*}$ for flexible and reconfigurable automation and are now being marketed at industrial materialhandling exhibitions as useful tools [2, 3]. Typical applications currently being considered for mobile manipulators are:i) unloading trucks [4], ii) bagged-goods (e.g., dog food bags) handling, iii) conveyer loading/unloading, iv) picking canned and boxed goods from shelves in supermarkets, and v) delivering, placing, and manipulating semiconductor wafer pods within wafer fabrication facilities [5]. The first four applications have looser constraints on the mobile manipulator pose (position

\footnotetext{
* Disclaimer: NIST does not endorse products discussed within this paper nor manufacturers of these products. Products mentioned are for information purposes only and are not expressed as an endorsement for them or their manufacturer.
} 
and orientation) and do not require precise alignment with the workspace. Vision is integrated into these systems to position a vacuum gripper to pick up the box, bag, or metal can in the manipulator's workspace. However, the last application, wafer pod manipulation, and other assembly-type operations, (e.g., peg-in-hole),require a much higher tolerance pose capability from the mobile manipulator.

The first four applications have looser constraints on position and orientation (pose) of the mobile manipulator; and, they do not require precise alignment with the workspace. To enhance the likelihood of success, vision is integrated into these systems so that they position a vacuum gripper to pick up the box, bag, or metal can. However, the last application, which targets waferpod manipulation and other assembly-type operations including "peg-inhole",has much tighter constraints onthe pose capability of the mobile manipulator.

The National Institute of Standards and Technology (NIST), Robotic Systems for Smart Manufacturing Program [6] is currently researching bothautomatic guided vehicle $(\mathrm{AGV}) /$ mobile manipulator performance and vision performance standards[7]. The Program develops and deploys advances in measurement science by improving performances of robotic systems to achieve dynamic production for assembly-centric manufacturing.

Assembly operations performed by a mobile manipulator [8] require accurate registration to the workpiece. We view 'registration' as instrument (e.g., mobile manipulator) comparison to a workpiece (e.g., artifact),or sometimes called a workstation, and 'calibration' as instrument (e.g., camera) adjustment or output correlation of the instrument readings with its known accuracy. These two terms are sometimes interchanged in the literature. Various registration methods have been researched, including:

- Quick Reference (QR) codes [8] combined with calibrated vision [9] tracking error: under $20 \mathrm{~mm}$, maximum errors: $45 \mathrm{~mm}$ at the largest camera-target distance.

- QR codes for mobile robot registrationand end effector error [10] maximum positional repeatability: $1.1 \mathrm{~mm}$ (one point) to $4.0 \mathrm{~mm}$ (multiple points).

- High-precision calibration - average errorsbased on the Tsai hand-eye calibration combined with a high-speed calibration - average errors: \pm 0.1 $\mathrm{mm}$ and $\pm 0.1^{\circ}$-based on a combination of laser triangulation and image processing [11]. 
- Constrained manipulator endpoint to a single contact point while executing manipulator motion where manipulator joint angles are read to develop a calibration model [12].

- Touch probing using peg-in-hole and particle filter solutions [13, 14].

This paper describes three alternative methods for registering mobile manipulators to a workpiece. The first builds upon [10] from Aalborg University where QR codes were used in combination with vision processing. The second and third use'fine' and 'bisect' search methods using a laser retroreflector to determine fiducial location with respect to the mobile manipulator. Experiments and experimental results are then described for each of these calibration alternatives.

\section{Registration Methods}

Registration of a mobile manipulator with a workpiece can be performed using a number of techniques, as briefly described in the introduction. If the accuracy requirements of the task are low, then simple navigation of the base into the desired pose may be adequate. However, we assume the accuracy requirements are greater than the base accuracy and more information is required for a suitable transformation between the manipulatorand workpiece coordinate systems. The following subsections describe three non-contact methods tested at NIST for registering a mobile manipulator to a workpiece detection of QR codes using vision and two search methods using a laser retroreflector and reflective fiducials. Future research may combine the registration methods by using a laser spotdetection method as described in [16 and 17].

\subsection{Visual Fiducials}

Visual fiducial systems allow for 6 degrees-of-freedom (6DOF) positional tracking of fiducial targets, or tags. Since these systems are well-developed, and can be implemented with open source software, inexpensive cameras, and virtually free printed targets, they have a number of advantages for use in robotics research and testing procedures for industrial robot evaluation and validation.

In this study, we reviewed fiducial systems commonly labeled as AR, or augmented reality. These systems include: ARTag, April tags, ARToolKit, and ALVAR [18]. We conducted experiments using the "A software Library for creating Virtual and Augmented Reality" or ALVAR version because of its integration with Robot Operating System (ROS). Integration with ROS allowed 
the use of ROS preprocessing, visualization, and message-passing facilities. Like the other systems, ALVAR uses rectangular black and white targets with a black outer square for location, and an internal matrix of squares that codes the identity of the target. Other fiducial targets used include standard cameracalibration targets (i.e., checkerboards), QR codes, and applicationspecific targets.

ALVAR and similar systems have advantages in flexibility and cost over other 6DOF tracking systems that may require more expensive and extensive installations.We need to understand robustness, working range and orientations, accuracy, and response timefor ALVAR use in testing procedures and standards. These needs do not have universal solutions since the system performance depends on implementation details. Robustness depends on occlusion and camera details; working range depends on target size, camera resolution, and camera focal length; response time depends on camera frame rate, resolution, and computer processing unit speed; and ultimate accuracy depends on all these factors, including target motion speed relative to frame rate.

\subsection{Fine and Bisect Search Methods}

A 'fine search' method was described in [17 and19] as it evolved; it is included in this paper to focus on the registration aspect. The method uses a laser retroreflector detector carried by the manipulator to detect reflective fiducials attached to the reconfigurable mobilemanipulator apparatus (RMMA). The RMMA is a metal plate with fiducial mount points at precise locations. The fiducial is a collimated reflector on a base that attaches to the RMMA.

A computeraideddesign model of paths and docking points was used by a vehicle control program to move the AGV from one docking pose to another near the RMMA. The vehiclecontrol program positioned the vehicle at various orientations with respect to the RMMA and the manipulator program corrected for vehicle pose allowing it to register with pre-taught targets using fine and 'bisect' search methods described here.

Two pairs offiducials were positioned at 1) two corners of a $457 \mathrm{~mm}$ square pattern of four fiducials and 2) at opposing points along a $305 \mathrm{~mm}$ diameter circle pattern of eight fiducials. The fine search originally used a circular search [17] and was tested only on the square pattern. However, it was quickly discovered that fiducial edges were detected causing a potential for the registration to be skewed andincreased search stepscaused the laser to pass over the fiducial without detecting it. 
In [19], a squaresearch method was tested. The 'square search' is a sequence of points in a spiral pattern on a square grid. Each step was $0.5 \mathrm{~mm}$, where the smaller step size and the use of $1 \mathrm{~mm}$ and $2 \mathrm{~mm}$ fiducials minimized previous issues. Figure 2 (a) shows a graphic of the square search method and Figure 2 (b) shows the RMMA (black table). The gray housings each include a camera iris that is used to change the fiducial detection diameter. This method works relatively well for aligning the mobile manipulator with the workpiece. However, errors in the mobile basepose measurement can cause a lengthy initial registration fine search.

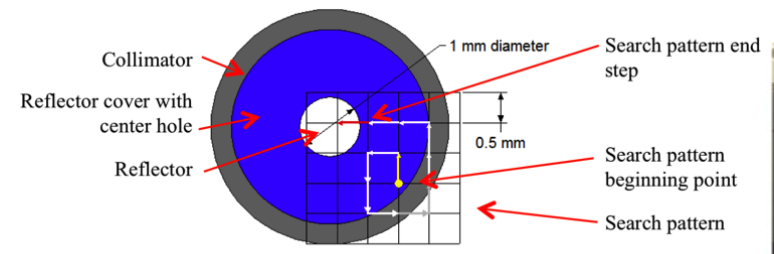

a

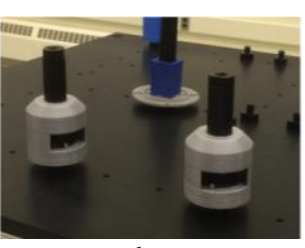

b

Figure 2: (a) Example square step search pattern drawing. The pattern begins with the yellow arrow dotted end and ends when the reflector is detected with the red arrow search step, (b) registration fiducial mechanisms attached to the RMMA and used to change the fiducial diameter.

For the bisect method, the detection of two,relatively large (42 $\mathrm{mm}$ diameter) reflectorswas performed before the fine search method. All reflectors were the same type micro-reflector. After detecting the large reflector, a bisecting search pattern determined the center of the reflector with $0.5 \mathrm{~mm}$ steps along relative $\mathrm{X}$ - and $\mathrm{Y}$ - axes to the manipulator base.

The reflector diameter was largeenough that no initial search was required to locate them, despite the measured maximum $13.3 \mathrm{~mm}$ error in the mobile base pose. Figure 3 (a) shows a concept drawing of the bisect method and Figure 3 (b) shows the mobile manipulator positioned next to the RMMA, the

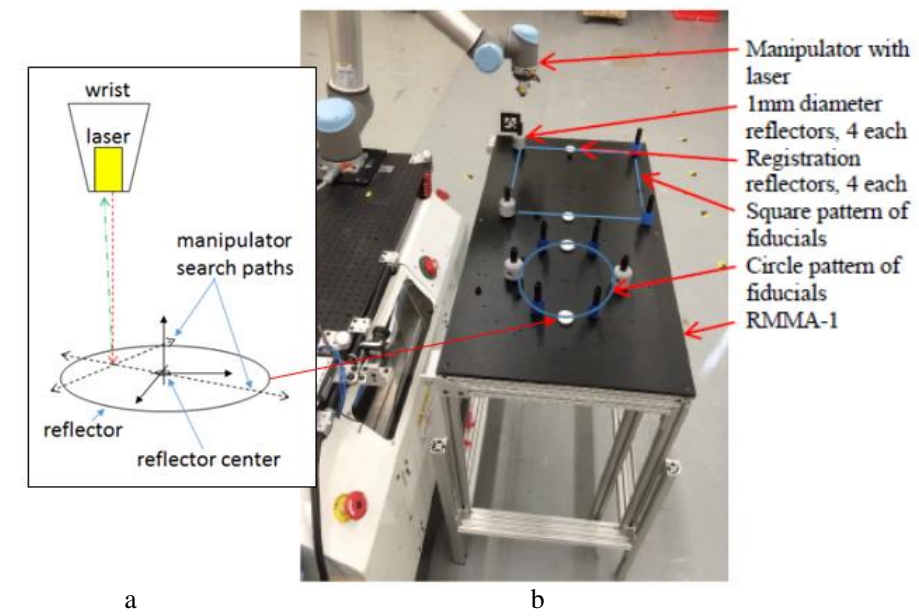

Figure 3 (a) Bisection search concept, (b) the mobile manipulator positioned next to the RMMA, the RMMA square and circle patterns, and the large reflectors within each pattern. 
RMMA square and circle patterns, and the large reflectors within each pattern. Once the center of the large reflector was located, the manipulator began a fine search of the $2 \mathrm{~mm}$ fiducials using the square search method.

\section{Experiments and Results}

\subsection{Visual Fiducials}

To evaluate and validate the use of visual fiducial targets, we conducted two sets of experiments using the ALVAR implementation and a $17 \mathrm{~mm}$ machine vision camera with a resolution of 1296 pixels x 964 pixels and a fixed $4.5 \mathrm{~mm}$ focal length lens.

The first set of experiments looked at the static repeatability of the ALVAR system when the target was moved to static positions by a pan-tilt mechanism. A $200 \mathrm{~mm} \times 200 \mathrm{~mm}$ target was mounted on a pan-tilt unit. For the experiments, the pan-tilt was moved systematically throughout its range and allowed to settle before static measurements. The camera viewed the target from a separation distance of $800 \mathrm{~mm}$ to $1000 \mathrm{~mm}$ as the target was systematically moved to 26 positions of differing tiltandpan. For each position, 306 measurements were taken over $30 \mathrm{~s}$. We calculated the root-mean-square deviation (RMSD) of the measurements to see if ALVAR gave consistent results. Repeatable measurements indicate systemic biases can be corrected by calibration.

We found that the maximum difference from the mean in any one position in any dimension was $0.8 \mathrm{~mm}$ (along the Z-axis), and the maximum angular error (in angle axis representation) was $0.18^{\circ}$. Each individual measurement was single shot with no averaging or filtering across measurements. From initial results of sub-millimeter repeatability in position, and fractionalangular repeatability, we judge that the basic capabilities of ALVAR are adequate as a subsystem in workpiece registration.

In the second set of visual fiducial experiments, we integrated ALVAR with the systems on an AGV docking with the RMMA. Spacing between the RMMA square and circle patterns was $508 \mathrm{~mm}$. The camera was onboard the AGV, repeatedly measured the $\mathrm{AGV}$ positioning at the square and circle patterns, and communicated the position to the robot controller.

\subsection{Fine and Bisect Search methods}

The RMMA was setup as shown in Figure 2 (b) for the circle pattern with 1 $\mathrm{mm}$ diameter registration fiducials. The circle used $1 \mathrm{~mm}$ diameter fiducials and the square used $3 \mathrm{~mm}$ diameter fiducials. The $3 \mathrm{~mm}$ fiducials were hypothesized 
to achieve faster registration although this was not the case.The AGV control program moved the AGV from a home position away from the RMMA to the first posepre-determined by the AGV control program. Upon completion of the pattern detection for the first pose, the AGV moved to the second pose, and so forth.Only the first six vehicle poses were completed for the 'fine search'method due to the long registration time. The $3 \mathrm{~mm}$ fiducial had the highest average number of search steps at 776 causing an average search time at approximately $360 \mathrm{~s}$ with a maximum of $893 \mathrm{~s}$. The root-mean-square deviation (RMSD) from the mean was 869 steps (403 s).

The 'bisect search' method experiment consisted oflocating the mobile manipulator in the same manner as in the fine search method. The RMMA was setup as shown in Figure 2 (b) with the circle and square patterns both using 42 $\mathrm{mm}$ diameter registration fiducials. After setup, the experiment was run for all 10 different mobile manipulator poses and repeated five times for a total of 50 poses. The results shown in Table linclude only the detection of the first $2 \mathrm{~mm}$ reflector for each pattern after bisect registration. The bottom of Table 1shows a summary of all tests averaged over the 50 measurements and includes the average number of steps for the $2 \mathrm{~mm}$ reflectors and shows the RMSD from the mean.

Table 1: Mobile manipulator registering to theRMMA using the bisectsearch method.

\begin{tabular}{|c|c|c|c|c|}
\hline AGV Position Number & Pose Angle & Pattern & $\begin{array}{c}\text { Average num. of search } \\
\text { steps to register }\end{array}$ & $\begin{array}{c}\text { Total bisect + fine search } \\
\text { time to register (s) }\end{array}$ \\
\hline 1 & $90^{\circ}$ & circle & 0 & 86 \\
\hline 2 & $315^{\circ}$ & square & 6 & 89 \\
\hline 3 & $0^{\circ}$ & circle & 0 & 86 \\
\hline 4 & $0^{\circ}$ & square & 0 & 86 \\
\hline 5 & $45^{\circ}$ & circle & 0 & 86 \\
\hline 6 & $90^{\circ}$ & square & 0 & 86 \\
\hline 7 & $135^{\circ}$ & circle & 0 & 86 \\
\hline 8 & $225^{\circ}$ & square & 0 & 86 \\
\hline 9 & $270^{\circ}$ & circle & 0 & 92 \\
\hline 10 & $270^{\circ}$ & square & 12 & $3.8 / 1.8$ \\
\hline
\end{tabular}




\section{Conclusions}

Experimental results reported for visual fiducials are consistent with the various registration methods from the literature. Under optimal conditions, we estimated repeatability of a visual fiducial at under $1 \mathrm{~mm}$ and $0.2^{\circ}$ from a single image. Given other elements in the system, including calibration of camera-tobase, and base-to-arm, and the propagation of error, we would expect total error for the system to be higher.

The finesearchmethod experiments resulted in a high number of search steps and time (average steps: 776, average/maximum time: $360 \mathrm{~s} / 893 \mathrm{~s}$ ) to register the mobile manipulator. When using the bisect method prior to the fine search, the total bisect plus fine search steps/time was a maximum of 184 steps/86 s or nearly $90 \%$ less time than using only the fine search method. Larger bisect search steps, among many other improvements, could be used although would increase the number of registration search steps on the $1 \mathrm{~mm}$ or $2 \mathrm{~mm}$ fiducials to a potentially unknown amount. Future registration tests will combine the visual fiducial with the search methods to minimize the search time.

\section{References}

1. ASTM Committee F45, WK48955working document, Navigation: Defined Space, April 2016.

2. ProMat 2015, Material Handling Ind. of America, Chicago, IL, March 2015.

3. Modex 2016, Material Handling Ind. of America, Atlanta, GA, April 2016

4. "Yaskawa Motoman MH80 robot unloading trucks - Wynright Corp.," http://www.youtube.com/watch?v=8wngL0BnF_4, June 2013.

5. Adept Lynx Handler-Semi, http://www.adept.com/products/mobile-robots/mobiletransporters/handler-semi/general

6. National Institute of Standards and Technology, Robotic Systems for Smart Manuf. Program, http://www.nist.gov/el/isd/ms/rssm.cfm, April 2016.

7. ASTM E2919-14, http://www.astm.org/Standards/E2919.htm, April 2016.

8. R. Andersen, O. Madsen, T. Moeslund, "Hand-Eye of Depth Cameras based on Planar Surfaces", Abstract from Int'l Workshop on Intell. Robot Assistants, Padova, Italy, 2014.

9. B. Hamner, S. Koterba, J. Shi, R. Simmons, S. Singh, "An autonomous mobile manipulator for assembly tasks" Auton. Robot 28: 131-149 (2010).

10. R. Andersen, J. Damgaard, O. Madsen, T. Moeslund, "Fast calibration of industrial mobile robots to workstations using QR codes." 44th Int'l Symp. Robotics (ISR), pp. 1-6. IEEE, 2013.

11. M. Hvilshøj, et al., "Calibration techniques for industrial mobile manipulators: Theoretical configurations and best practices." Robotics (ISR), 2010 41st International Symposium on and 2010 6th German Conference on Robotics (ROBOTIK). VDE, 2010. 
12. M. Meggiolaro, G. Scriffignano, S. Dubowsky, "Manipulator Calibration Using A Single Endpoint Contact Constraint", Proceedings of DETC2000: 2000 ASME Design Engineering Technical Conference, DETC2000/MECH-14129, Baltimore, MD, September 2000.

13. S. Chhatpar, M. Branicky, "Localization for Robotic Assemblies Using Probing and Particle Filtering", Proc. of the 2005 IEEE/ASME Int'l Conf. on Advanced Intelligent Mechatronics, Monterey, CA, USA, 24-28 July 2005.

14. Y. Taguchi, T. Marks, J. Hershey, "Entropy-Based Motion Selection for TouchBased Registration Using Rao-Blackwellized Particle Filtering”, TR2011-067, September 2011.

15. M. Mesko, S. Toth, "Laser Spot Detection", Journal of Information, Control and Management Systems, 11, No. 1 (2013).

16. A. Krstinic, K. Skein, I. Milatic, "Laser Spot Tracking Based On Modified Circular Hough Transform and Motion Pattern Analysis", Sensors, 14, 20112-20133; ISSN 1424-8220, (2014).

17. R. Bostelman, T. Hong, J. Marvel, "Performance Measurement of Mobile Manipulators", SPIE 2015, Baltimore, MD, April 2015.

18. N. Macias, J. Wen, "Vision guided robotic block stacking", IEEE/RSJ Int'l Conf. Intelligent Robots and Systems (IROS 2014), pp. 779-784, 2014.

19. R. Bostelman, T. Hong, S. Legowik, "Mobile Robot and Mobile Manipulator Research Towards ASTM Standards Development", SPIE 2016, Baltimore, MD, April 2016. 